\title{
Auswirkungen vorschulischer Kinderbetreuung auf die Bildungschancen von Migrantenkindern ${ }^{1}$
}

\author{
Von Rolf Becker und Patricia Tremel
}

\begin{abstract}
Zusammenfassung: Vor dem Hintergrund der Nachteile von Migranten im deutschen Bildungssystem stellt sich die Frage nach geeigneten Maßnahmen, Chancengerechtigkeit beim Bildungszugang und Bildungserwerb herzustellen. In der empirischen Studie wird mit Daten des Sozio-ökonomischen Panels im Längsschnitt untersucht, ob die nachteilige Situation von Migranten im Schulsystem durch vorschulische Betreuung, Erziehung und Bildung reduziert werden kann. Die Befunde zu den Bildungseffekten vorschulischer Betreuung sind für den Zeitraum von 1984 bis 2003 ambivalent. Zwar verbessern sich die Bildungschancen von Migrantenkindern infolge vorschulischer Kinderbetreuung, aber selbst dann ziehen sie bei den Bildungschancen nur mit den einheimischen Kindern gleich, die nicht an vorschulischer Betreuung partizipiert haben. Insgesamt weisen sie dann die gleichen Bildungschancen wie deutsche Arbeiterkinder auf.
\end{abstract}

\section{Einleitung}

In der Bildungsforschung werden ethnische Differenzierung im Bildungssystem und Anbindung von Bildungschancen an den Migrationsstatus oder an die Staatsangehörigkeit als zunehmend wichtige Ungleichheitsdimensionen angesehen (Alba et al. 1994; Nauck et al. 1998; Esser 2001; Kristen 2002; Diefenbach 2004). Defizitäre Bildungschancen von Migranten sowie Chancenungerechtigkeit, wonach neben einheimischen Schulkindern aus unteren Sozialschichten auch den meisten Migrantengruppen faire Bildungschancen vorenthalten werden, stellen in Deutschland kein randständiges Phänomen dar (Nauck 1994; Steinbach und Nauck 2004). Trotz vielfältiger bildungs- und sozialpolitischer Bemühungen bestehen bei gestiegener Bildungsbeteiligung unter den Migranten und einiger Bildungserfolge in der Generationenabfolge immer noch deutliche Bildungsungleichheiten fort (Nauck und Diefenbach 1997; Gang und Zimmermann 2000; Diefenbach 2004; Becker und Lauterbach 2004). Beispielsweise werden Kommunikationsschwierigkeiten bei Migrantenkindern oder ihre mangelnde Sprachkenntnisse vielfach als Lernbehinderung interpretiert, so dass sie überproportional häufig in Sonderschulen für Lernbehinderte vertreten sind (Powell und Wagner 2001). Des Weiteren wechseln Migrantenkinder deutlich häufiger als einheimische Kinder von der Grundschule in die unteren Schullaufbahnen in der Sekundarstufe I und sind seltener in Gymnasien vertreten, dafür aber in der Hauptschule überrepräsentiert. Ebenso erhalten Migrantenkinder häufiger als einheimische Kinder eine Bildungsempfehlung für Haupt- oder Sonderschulen und überaus seltener eine Bildungsempfehlung für höhere Schullaufbahnen in der Sekundarstufe I, was zum großen Teil, aber nicht vollständig oder in jedem Fall, ihren Noten in Deutsch und Mathematik entspricht (Diefenbach 2004). Nicht zuletzt haben Ergebnisse von PISA 2000 für Deutschland ein hinlänglich bekanntes Faktum in der Öffentlichkeit wieder in Erinnerung gerufen: Sowohl Kinder und Jugendliche aus jüngst zugewanderten Migrantengruppen als auch Kinder von Migranten aus der zweiten und dritten Generation weisen in der Regel nicht nur schlechtere Lesekompetenzen als einheimische Jugendliche auf, sondern sind auch - mit großen Variationen zwischen den Nationalitäten und verglichen mit den Einheimischen - im bundesdeutschen Bildungssystem im Nachteil und beim Bildungserwerb möglicherweise benachteiligt (Baumert et al. 2001).

1) Für wertvolle Hinweise danken wir Anna Hecken, Andreas Hadjar, Wolfgang Lauterbach und den anonymen Gutachtern.

Soziale Welt 57 (2006), S. $397-418$ 
In der Bildungspolitik wird daher, um mehr Chancengerechtigkeit bei der Einschulung herzustellen, frühe vorschulische Förderung der Kinder von Migranten - die Zielgruppe vorschulischer Sprachförderung - in Kindertageseinrichtungen gefordert (z.B. Arbeitsstab »Forum Bildung « 2001). Ob Kindergärten und Vorschulen in Deutschland derzeit zu diesem Ziel beitragen, kann nur anhand empirischer Überprüfungen von Bildungseffekten vorschulischer Kinderbetreuung beurteilt werden (vgl. Büchel et al. 1997; Becker und Lauterbach 2004). Daher wird im vorliegenden Beitrag folgende Fragestellung untersucht: In welchem Ausmaß fördern vorschulische Bildungsmaßnahmen die Bildungschancen von Migrantenkindern unterschiedlicher Nationalitäten im deutschen Schulsystem? Inwieweit können die Migrantenkinder dann bei den Bildungschancen zu den einheimischen Schulkindern aufschließen?

Im zweiten Abschnitt des Beitrags wird der Forschungsstand diskutiert, danach werden relevante Gründe angeführt, warum positive Bildungseffekte vorschulischer Betreuung zu erwarten sind und schließlich theoriegeleitet Hypothesen abgeleitet. Für empirische Analysen werden Längsschnittdaten des Sozio-ökonomischen Panels (SOEP) herangezogen, die gemeinsam mit den abhängigen und erklärenden Variablen im dritten Abschnitt dargestellt werden. Die Darstellung zentraler empirischer Befunde erfolgt im vierten Abschnitt, und im fünften Abschnitt werden die Ergebnisse diskutiert und Schlussfolgerungen gezogen.

\section{Forschungsstand und theoretischer Hintergrund}

\section{Kurzer Überblick über den Forschungsstand}

Es gibt in der Zwischenzeit einige empirische Belege für positive Bildungseffekte vorschulischer Betreuung in Kindergarten und Vorschule (Becker und Lauterbach 2004; Spieß und Tietze 2002; Kreyenfeld et al. 2002; Büchel et al. 1997). ${ }^{2}$ Unter Verwendung von Längsschnittdaten des Sozio-ökonomischen Panels können Büchel, Spieß und Wagner (1997) für den Zeitraum von 1992 bis 1994 empirisch nachweisen, dass der Besuch vorschulischer Kinderbetreuungseinrichtungen (Kindergarten, Vorschulen etc.) einen förderlichen Einfluss auf den Bildungserfolg dieser Schulkinder hat. So haben Schulkinder mit Migrationshintergrund, die keine der vorschulischen Bildungseinrichtungen besucht haben, ein signifikant höheres Risiko, im Alter von 14 Jahren die Hauptschule zu besuchen, als die Migrantenkinder mit Kindergartenbesuch (Büchel et al. 1997, S. 536-537). Frühe Investitionen in die Ausbildung der Migranten führen nicht nur zu erwünschten Bildungserfolgen, sondern scheinen ein wirksames Instrumentarium zu sein, ihre Sozialintegration zu fördern sowie über kompensatorische Erziehung und Bildung deren herkunftsbedingten Nachteile bei den Bildungschancen auszugleichen (Büchel et al. 1997, S. 537-538). In einer weiterführenden Analyse mit den gleichen Daten können Becker und Lauterbach (2004) die Befunde von Büchel, Spieß und Wagner (1997) weitgehend replizieren. Auch bei Kontrolle sozialer Selektivität bei der Partizipation vorschulischer Kinderbetreuung können für einen längeren Zeitraum von 1992 bis 2000 positive Bildungseffekte vorschulischer Kinderbetreuung nachgewiesen werden.

In vielen Fällen kann davon ausgegangen werden, dass aufgrund ungünstiger Sozialisationsbedingungen im Elternhaus, die bei Migranten durch die Umstände und den Zeitpunkt der Migration verschärft werden können, Migrantenkinder sowohl bei den Startchancen als auch bei der Entwicklung schulischer Leistungen im Nachteil sind. Sie werden daher nur er-

2) So stellen beispielsweise Bos et al. (2003, S. 129) bei den ersten Auswertungen der PIRLS 2001-Studie - einem internationalen Vergleich von Schülerleistungen am Ende der vierten Jahrgangsstufe, der in Deutschland unter der Bezeichnung IGLU für Internationale Grundschulleseuntersuchung bekannt ist - fest, dass gerade Kinder aus Arbeiterschichten bessere Lesekompetenzen aufweisen, wenn sie mehr als ein Jahr den Kindergarten besucht haben. 
schwert die Schulnoten erwerben, die zum Übergang in die weiterführenden Schullaufbahnen in der Sekundarstufe I berechtigen. Weil Schulleistungen an soziale und nationale Herkunft gekoppelt sind, wird eine Form der intergenerationalen Bildungsvererbung in Gang gesetzt, die das Prinzip der Chancengleichheit aushebelt, wobei neben Kindern aus unteren Sozialschichten vor allem Migrantenkinder die »Hauptopfer« von Selektionsmechanismen im Bildungssystem sind. Des Weiteren bringt die Migration eine zusätzliche Schlechterstellung zugewanderter Kinder mit sich, wenn sie die Sprache des Aufnahmelandes kaum oder nicht beherrschen, in Zusammenhang mit einem höheren Einreisealter quer in die Schule des Aufnahmelandes einsteigen oder die vorschulischen Betreuungs- und Fördereinrichtungen des Aufnahmelandes nicht besucht haben. Disparitäten der Lernvoraussetzungen und Startchancen zwischen Migrantenkindern und einheimischen Kindern können eine Folge dessen sein.

\section{Theoretisch erwartete Bildungseffekte vorschulischer Erziehung, Betreuung und Bil- dung}

Vorschulische Erziehung impliziert grundsätzlich einen positiven Effekt auf die Kompetenzentwicklung und den weiteren Bildungsverlauf von Kindern. Bis zum Eintritt in die formalen Bildungsinstitutionen ist es vor allem die Familie, die das Lernverhalten des Kindes prägt. Vorschulische Erziehung von Seiten einer Institution verspricht zum einen eine zusätzliche Förderung des Kindes und zum anderen möglicherweise einen ungünstige sozialisatorische Einflüsse ausgleichenden Effekt auf die Lernfortschritte (Schütz und Wössmann 2005, S. 19). Im Sinne der Chancengleichheit wird erhofft, dass vorschulische Betreuung, Erziehung und Bildung mögliche Defizite der Herkunftsfamilie ausgleichen und dass das frühe Lernen die Effektivität des späten Lernens erleichtert (Schütz und Wössman 2005, S. 21). So konstatiert Heckhausen (1969), dass in den drei Jahren vor der Einschulung die entscheidende Grundlage für die intellektuelle Leistungsfähigkeit der Schüler gebildet wird. Demnach würde eine vorschulische Betreuung die kognitive Entwicklung des Kindes maßgeblich beeinflussen. Durch Kindergarten und Vorschule kann die Entwicklung des Kindes gezielt beeinflusst werden, indem das Anregungspotenzial der Elternhäuser in wichtigen Punkten ergänzt wird. Insbesondere untere Sozialschichten und jene Sozialgruppen, deren soziokulturelles Milieu »die Entfaltung der kindlichen Potenzialitäten schon in den ersten Jahren vermindert«, könnten die Defizite durch den Besuch vorschulischer Institutionen ausgleichen (vgl. Heckhausen 1969, S. 207). Vorschulische Betreuung kann bei einer gezielten pädagogischen Förderung sozial benachteiligter Kinder durchaus geeignet sein, den vergleichsweise ungünstigen sozialisatorischen Einfluss von Elternhäusern in den unteren Sozialschichten oder mit Migrationshintergrund auf den Bildungserfolg abzuschwächen, der zu ungleichen Startchancen beim Bildungserwerb und damit langfristig zu Bildungsungleichheiten führt. Sinnvoll ist daher, eine relative Chancengleichheit bereits bei der Einschulung herzustellen, bevor der Wettbewerb im Bildungssystem beginnt. Insbesondere für Kinder aus Elternhäusern, die entweder über wenig Einkommen und Humankapital verfügen oder in geringem Maße mit den Bildungsmöglichkeiten vertraut sind, könnten frühe Erfahrungen mit schulischen Einrichtungen und Abläufen sowie mit dem Alltag in solchen Institutionen und den signifikant Anderen wie etwa Lehrpersonal eine unterstützende Funktion bei der kritischen Phase des Schuleintritts haben. Für den weiteren Bildungsverlauf kann die frühe Bildungsentscheidung der Eltern, ihr Kind vorschulisch betreuen zu lassen, langfristige Konsequenzen haben. Doch nicht zuletzt ist diese Entscheidung auch mit ökonomischen Aspekten verbunden. Migranten verfügen in der Regel über geringeres Finanz- und Humankapital, das sie für Investitionen in die Bildung ihrer Kinder mobilisieren können (Nauck et al. 1998; Diefenbach und Nauck 1997). So entscheiden sie sich im weiteren Bildungsverlauf - bei Berücksichtigung der schulischen Leistungen ihrer Kinder und den daraus resultierenden Erwartungen über den realisierbaren Bildungserfolg - eher als Einheimische für die kürzeren und weniger kostenintensiven Bil- 
dungsgänge. ${ }^{3}$ Demnach bedeutet schon eine derartige vorschulische Investition in Bildung für Migranten in vielen Fällen mehr Aufwand als für einheimische Eltern. Doch gerade bei Kindern aus nachteiligen sozioökonomischen Verhältnissen sollen mit Hilfe vorschulischer Betreuung die unter Umständen vergleichsweise mangelhaften kognitiven Fähigkeiten und defizitäre Sprachfähigkeiten ausgeglichen werden. Demnach müssten Bildungseffekte vorschulischer Bildung und Betreuung in systematischer Weise die ungünstigen Auswirkungen der nationalen und sozialen Herkunft auf die schulischen Leistungen und elterlichen Bildungsentscheidungen kompensieren, damit die Bildungschancen nicht mehr vom Migrationsstatus abhängen (vgl. Kristen 2002). Somit wäre es zu erwarten, dass die vorschulische Betreuung gerade für Migranten eine positive Wirkung haben könnte und entsprechend die Bildungschancen der Migrantenkinder deutlich erhöhen würde.

\section{Abbildung 1: Heuristisches Modell zur Erklärung von Bildungsungleichheiten}

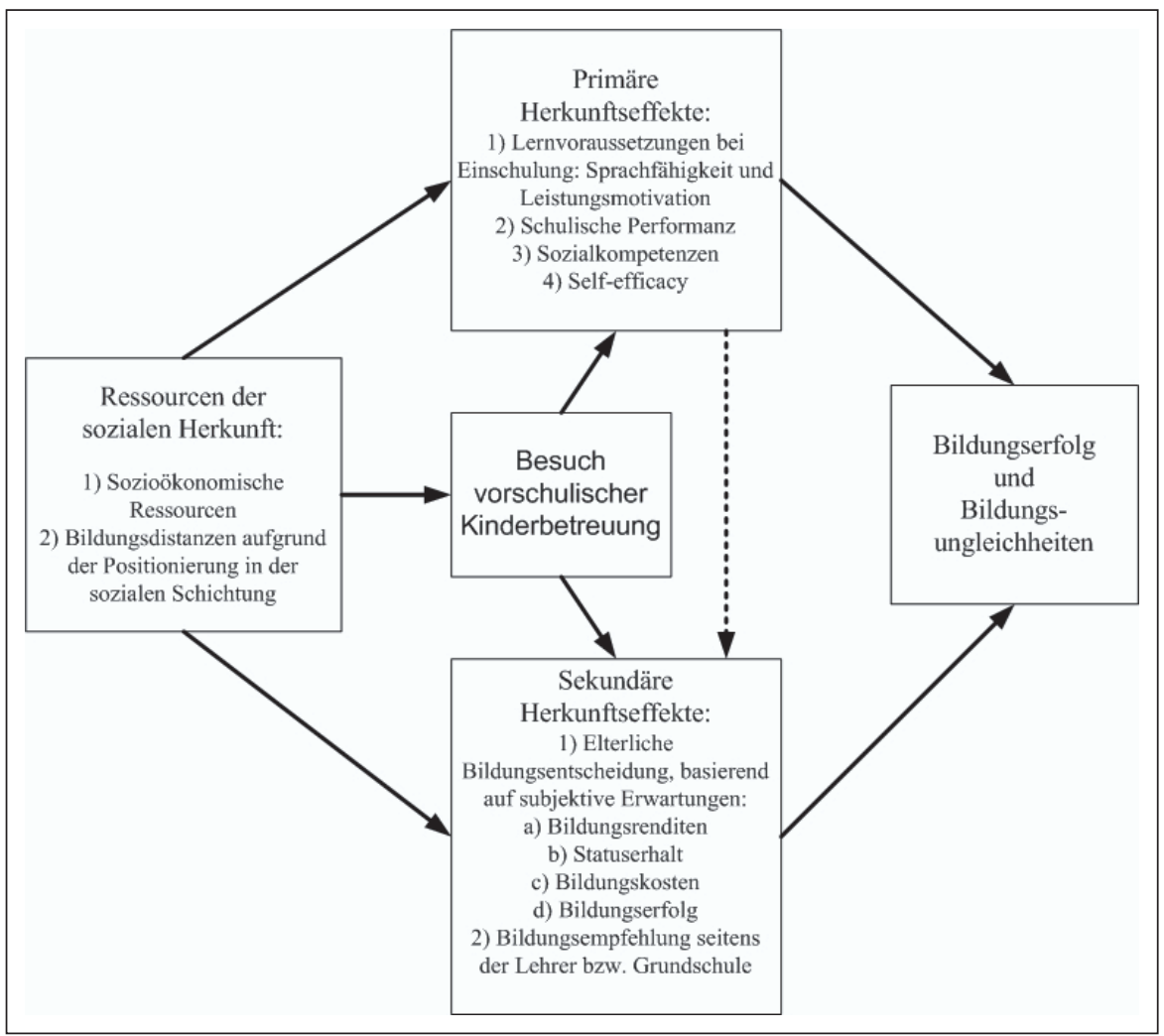

3) Die Wahl der Schullaufbahn am Ende der Grundschulzeit wird von den Eltern als eine nachwirkende Weichenstellung für den Lebensverlauf von Kindern angesehen. Die erwarteten Lebenschancen werden an dieser Sichtweise ausgerichtet. Ziel dieser Lebensplanung ist vor allem bei der autochthonen Bevölkerung die Erhaltung des sozialen Status in der Generationenfolge, während bei der Migrationsbevölkerung vielfach eher die vorteilhafte, auf Einkommenserzielung abstellende Platzierung der Kinder in die Arbeitsmärkte im Vordergrund steht (Nauck 1994). Demnach gilt ein Streben nach maximaler Schulbildung als Grundlage elterlicher Bildungsaspirationen. Allerdings können diese nur dann realisiert werden, wenn die Eltern über entsprechende Ressourcen verfügen, um längere Ausbildungen finanzieren zu können. 
Kurz- und langfristige Effekte auf schulische Leistungen und Kompetenzentwicklung können sowohl die elterliche Bildungsaspirationen und -entscheidungen als auch die gezielte Förderung und Leistungsbewertung durch Lehrer in positiver Weise zu Gunsten sozial benachteiligter Kinder beeinflussen (siehe Abbildung 1). So liegen empirische Befunde aus US-amerikanischen Studien dafür vor, dass der Besuch vorschulischer Einrichtungen - indirekt über günstige schulische Performanz und Schulleistungen sowie direkt über Attribution von Bildungsmotivation und Leistungswille - die Beurteilung durch Lehrpersonen beeinflussen kann. Im Falle positiver sozialisatorischer und kognitiver Effekte werden positive Erwartungen bei Eltern und Lehrern erweckt, so dass sie die Kinder fördern und zur höheren Bildung ermutigen (Entwisle 1995, S. 135). Dadurch gesteigertes Vertrauen in die Leistungsfähigkeit der Kinder erhöht wiederum deren schulische Performanz (Entwisle 1995, S. 139). Für Deutschland wären kompensatorische Auswirkungen auf Leistungsbewertungen und somit eine Reduktion der sozialen Selektivität von Bildungsempfehlung der abgebenden Grundschule zu erwarten.

Welche Wirkung hat nun die vorschulische Betreuung von Kindern allgemein und von Migrantenkindern im Speziellen? Die bisherigen Ausführungen haben darauf verwiesen, dass vorschulische Betreuung soziale Ungleichheiten von Bildungschancen ausgleichen soll und in Hinblick auf die bevorstehende Schulzeit eine Verbesserung der kognitiven Fähigkeiten der Individuen mit sich bringen könnte. Es wird angenommen, dass soziale Ungleichheiten von Bildungschancen ein aggregiertes Ergebnis des Zusammenwirkens primärer und sekundärer Herkunftseffekte ist (siehe Abbildung 1). ${ }^{4}$ Die Entscheidung der Eltern zu einem Kindergarten- oder Vorschulbesuch des Kindes steht zum einen in Abhängigkeit von den jeweiligen Herkunftseffekten und zum anderen beeinflusst diese Entscheidung vermutlich auch den weiteren Bildungswerdegang des Kindes. Entsprechend lassen sich Bildungsungleichheiten durch derartiges Entscheidungsverhalten erklären.

Aus diesen theoretischen Überlegungen ergeben sich folgende Hypothesen: Generell werden für Jugendliche mit Migrationsstatus positive Bildungseffekte vorschulischer Bildung und Betreuung erwartet. (1) Aufgrund von vorschulischer Bildung in Kindergärten und Vorschulen haben die Migrantenkinder günstigere Chancen, auf die höheren Schullaufbahnen in der Sekundarstufe I zu wechseln, als diejenigen unter ihnen ohne jegliche vorschulische Bildung. (2) Ferner ist zu erwarten, dass sie dadurch ihre im Vergleich zu einheimischen Schulkindern deutlichen Bildungsabstände erheblich verringern können.

\section{Datenbasis und Variablen}

\section{Datenbasis}

Um Bildungseffekte vorschulischer Kinderbetreuung und Bildung aufdecken zu können, benötigt man Längsschnittdaten. Daher greifen wir auf das Sozio-ökonomische Panel zurück (SOEP Group 2001). Im Rahmen dieses Panels werden seit 1984 jährlich die gleichen priva-

4) Primären Herkunftseffekte sind die kognitiven Vor- und Nachteile eines Individuums, die in Abhängigkeit von der Zugehörigkeit einer Sozialschicht unterschiedlich stark ausgeprägt sein können. So tragen diese primären Herkunftseffekte als eine weitere Ursache für sozial selektive Bildungschancen langfristig zur Festschreibung dauerhafter Bildungsungleichheiten über Selektion und Sortierung auf die einzelnen Bildungslaufbahnen entsprechend der Schulleistungen bei. Sekundäre Herkunftseffekte sind die elterlichen Bildungsentscheidungen, welche in Abhängigkeit von vorhandenen ökonomischen Ressourcen der Herkunftsfamilie getroffen werden. Diese sekundären Effekte der sozialen und auch nationalen Herkunft, die auf rationalen, sich an ihren Möglichkeiten orientierenden Entscheidungen der Eltern beruhen, sind eine zentrale Ursache für sozial selektive Bildungschancen (Becker 2000; Esser 1999; Breen und Goldthorpe 1997; Boudon 1974). 
ten Haushalte und ihre Mitglieder in der Bundesrepublik Deutschland wiederholt befragt. In den privaten Haushalten werden alle Personen interviewt, die älter als 16 Jahre sind. Informationen über Kinder unter 16 Jahren werden über den Haushaltsvorstand festgehalten. Im Jahre 1990 wurde die bislang auf die Bundesrepublik beschränkte Erhebung von Quer- und Längsschnittinformationen auf das Gebiet der ehemaligen DDR ausgeweitet. Zusätzlich zu den Ost- und Westdeutschen enthält das SOEP auch Informationen über Migranten unterschiedlicher Nationalitäten, so dass Bildungschancen von Migrantenkindern im bundesdeutschen Bildungssystem im Vergleich zu einheimischen Schulkindern untersucht werden können. Wegen unterschiedlicher Voraussetzungen - für die Migranten liegt beispielsweise ein over-sampling vor - und Beobachtungszeitfenster - etwa die Zeit vor und nach der deutschen Einheit - werden die Analysen für die drei Bevölkerungsgruppen - Westdeutsche, Ostdeutsche und Migranten - separat vorgenommen. Bei den Migranten werden aufgrund der Sonderbedingungen der gesellschaftlichen Transformation in Ostdeutschland und ihres geringen Anteils in der ostdeutschen Bevölkerung nur diejenigen im Westen Deutschlands berücksichtigt.

Für den Zugang zur vorschulischen Kinderbetreuung untersuchen wir für den Zeitraum von 1984 bis 2003 die Kinder im Alter von drei bis sieben Jahren, bevor sie eingeschult werden. Aus logischen Gründen betrachten wir für die Auswirkung vorschulischer Kinderbetreuung auf die Verteilung auf die Schullaufbahnen in der Sekundarstufe I im Alter von 14 Jahren die soziale Selektivität vorschulischer Betreuung im Zeitraum von 1984 bis 1995 und den Übergang am Ende der Grundschulzeit - jeweils rund acht Jahre später - im Zeitraum von 1992 bis 2003. Im Unterschied zu einer vorhergehenden Untersuchung wird bei den Betreuungseffekten auf die Bildungschancen nicht nur der Besuch bzw. Nichtbesuch vorschulischer Kinderbetreuung im letzten Jahr, sondern jegliche Nutzung vorschulischer Betreuungseinrichtungen vor der Einschulung berücksichtigt (Becker und Lauterbach 2004). Schließlich werden die 14-jährigen Schulkinder ausgeschlossen, die andere Schulen (Integrierte Gesamtschulen, Schularten mit mehreren Bildungsgängen, Freie Waldorfschulen, Sonderschulen) als das Gymnasium oder die Haupt- und Realschule besuchen. Durchschnittlich bleibt dadurch rund ein Fünftel eines Jahrgangs unberücksichtigt.

\section{Abhängige und unabhängige Variablen}

Die erste abhängige Variable ist die Nutzung vorschulischer Kinderbetreuung in Kinderkrippen, Kindergärten, Kindertagesstätten und Vorschulen von drei- bis siebenjährigen Kindern. Abgesehen davon, dass bei den jeweiligen Befragungszeitpunkten die Form und Dauer der Betreuung in jeweils unterschiedlicher Weise erhoben wurde, wurde bis zur Panel-Welle im Jahre 1999 auch die Betreuung durch eine Tagesmutter einbezogen. Auf diese Schwierigkeit weisen Büchel, Spieß und Wagner (1997, S. 532) hin, da bei solch einer Betreuung im Unterschied zu den anderen Betreuungsmöglichkeiten der Bildungsaspekt eher sekundär sei. Da Kindergärten oder Vorklassen nicht durchgängig separat erfasst werden, gehen wir davon aus, dass die erste abhängige Variable auch alle kindergarten- und vorschulähnliche Einrichtungen mit einschließt. Eine detailliertere und über die einzelnen Zeitpunkte hinweg einheitliche Operationalisierung der vorschulischen Kinderbetreuung wäre wünschenswert gewesen, um differenzierte Aussagen über ihre Bildungseffekte treffen zu können. So wird im Folgenden recht grob danach unterschieden, ob ein Kind eine vorschulische Einrichtung besucht hat oder nicht. Aufgrund dessen gehen wir davon aus, dass damit diverse Formen vorschulischer Betreuung, Erziehung und Bildung erfasst werden und daher verwenden wir auch diesen Begriff, um Bildungseffekte von institutioneller Betreuung vor der Einschulung abzubilden.

Die zweite abhängige Variable ist für die 14-jährigen Schulkinder der Besuch des Gymnasiums, der Realschule oder der Hauptschule. Die Referenzkategorie ist der Übergang auf die 
Hauptschule. Alle anderen Schullaufbahnen wurden deswegen von der Analyse ausgeschlossen, weil der avisierte Abschluss eines Bildungszertifikats nicht eindeutig ist (z.B. Gesamtoder Waldorfschule) oder der Anteil der Bildungsgruppen für aussagekräftige Analysen zu klein ist (z.B. Sonder- oder Spezialschule).

Als erklärende Variablen für den sozial selektiven Besuch vorschulischer Einrichtungen wird neben dem Alter der Kinder auch die die historische Zeit (Periode) kontrolliert. Neben dem sozialen Wandel sollen dadurch auch Effekte eines gepoolten Datensatzes berücksichtigt werden. Des Weiteren wird das Bildungsniveau der Elternteile kontrolliert. Es wird als Kombination schulischer und beruflicher Abschlüsse operationalisiert und mit der durchschnittlichen Dauer bis zum Erwerb dieser Zertifikate gemessen. Zwar geht dadurch der ordinale Charakter erworbener Bildungszertifikate verloren, aber der Vorteil dieser Operationalisierung liegt in unserem Fall darin, dass bei multivariaten Analysen angesichts kleiner Fallzahlen nur eine geringere Zahl von Parametern geschätzt werden muss. Als außerordentlich ungünstig für unsere Fragestellung erweist es sich, dass mit den Daten des SOEP der Einfluss schulischer Leistungen sowie der Bildungsempfehlungen auf den Übergang in die Sekundarstufe I und damit eine wichtige Komponente der primären Herkunftseffekte und des Bildungserfolgs nicht kontrolliert werden kann (vgl. Becker 2003).

Als zusätzliche Messung der sozialen Herkunft des Kindes werden sowohl das Klassenstrukturschema von Erikson und Goldthorpe (1992) als auch der sozioökonomische Status des Haushaltsvorstandes, gemessen anhand des sozialen Prestiges nach Wegener (1988), herangezogen. Ebenso wird die Erwerbstätigkeit der Mutter als bedeutsam für die Nutzung außerhäuslicher Kinderbetreuung angesehen. Weitere sozialstrukturelle und sozioökonomische Strukturen des Haushaltes werden über die Anzahl der im Haushalt lebenden Personen (Haushaltsgröße) und mit dem verfügbaren Haushaltsnettoeinkommen gemessen.

Während die Erwerbstätigkeit der Mutter und der sozioökonomische Status des Elternhauses auch die berufliche Integration ausdrückt, wird die Qualität der deutschen Sprache der Elternteile (Selbsteinschätzung von » 1 « für »sehr gut« bis »5« für »kein Deutsch«) als Indikator für kulturelle Integration berücksichtigt. Für den Besuch einer der weiterführenden Schullaufbahnen nach der Grundschule wird zudem das Geschlecht des Kindes (Referenz: Mädchen) und der Besuch einer vorschulischen Einrichtung als erklärende Variable herangezogen. Die Nationalität des Haushaltsvorstandes bemisst trotz aller Schwierigkeiten bei der Abgrenzung des Migrationsstatus sowohl den Migrationshintergrund der Kinder als auch die nationale Heterogenität unter den Migranten. Entsprechend der Anlage des SOEP werden vornehmlich die türkische, (ex-)jugoslawische, griechische, italienische, spanische und deutsche Nationalität berücksichtigt (Referenz: andere Nationalitäten).

\section{Methodische Konsequenzen bei der Messung von Bildungseffekten}

Die empirische Überprüfung der Wirksamkeit von vorschulischer Bildung für spätere Bildungs- und Lebenschancen kann man durchaus als ein sozial- und bildungspolitisches Experiment ansehen. Liegen Umfragedaten vor, wird dieser Test statt in einem experimentellen eben in einem quasi-experimentellen Design vorgenommen (Cook und Campbell 1979). Dann treten bei der Beurteilung der Effektivität vorschulischer Kinderbetreuung methodische und statistische Schwierigkeiten auf, da keine zufällige Aufteilung von Untersuchungsund Kontrollgruppen erfolgt, wie dies für reine Experimente zwingend notwendig ist. Wenn aber die soziale Selektivität beim Zugang zur vorschulischen Bildung, also die systematische Aufteilung in die Untersuchungs- und Kontrollgruppen nach bestimmten Individualmerkmalen, nicht explizit kontrolliert wird, dann führen multivariate Analysen wegen eines »selectivity bias « zu verzerrten und daher wenig aussagekräftigen Ergebnissen. Deswegen verbietet es sich, in multiplen Regressionen die Nichtteilnehmer ohne jegliche Kontrolle ihrer sozialen 
Selektivität in der vorschulischen Kinderbetreuung als Kontrollgruppe heranzuziehen. ${ }^{5}$ Für multivariate Schätzungen von Bildungseffekten ist die Einbeziehung einer Dummy-Variablen, die indiziert, ob ein Kind an vorschulischer Bildung teilgenommen hat oder nicht, daher ausgeschlossen (Maddala 1978, S. 426). Dieses Evaluationsproblem kann allerdings durch die Kontrolle sozialer Selektivität bei der Nutzung vorschulischer Kinderbetreuung und Bildung vor der Einschulung zumindest teilweise gelöst werden (Lechner 1998).

Aus pragmatischen Gründen verwenden wir für die Kontrolle des Selektivitätsproblems das von Heckman (1979) vorgeschlagene Verfahren, das sich bereits in mehreren bildungssoziologischen Studien bewährt hat (vgl. Becker 2000, 2003). ${ }^{6}$ Bei der Ermittlung des Nutzens vorschulischer Kinderbetreuung für den weiteren Bildungsweg wird im ersten Schritt der Selektionsprozess beim Zugang zu vorschulischen Einrichtungen geschätzt. Die Schätzergebnisse für die sozial selektive Nutzung vorschulischer Kinderbetreuung, die so genannten inversen Mill's ratios (IMR), gehen im zweiten Schritt als instrumentelle Variable $\lambda$ (vorschulische Bildung) in die Schätzung der Nutzenfunktionen ein - eben in das Modell für den Übergang von der Primarstufe auf die weiterführenden Schullaufbahnen in der Sekundarstufe I. Die inversen Mill's Ratios (IMR) basieren auf Regressionsschätzungen für den Besuch vorschulischer Betreuungs- und Bildungseinrichtungen (Long 1997). So muss man bei der Verwendung des zweistufigen Verfahrens von Heckman (1979) wegen Multikollinearität darauf achten, dass nicht die gleichen erklärenden Variablen im Selektions- und im Effektmodell verwendet werden. In unserem Fall interpretieren wir die IMR als bedingte Wahrscheinlichkeit für die Nutzung vorschulischer Betreuungsangebote (Becker 2000). Damit ist es möglich, den kausalen Effekt vorschulischer Kinderbetreuung auf die Bildungschancen zu beurteilen.

\section{Empirische Befunde}

\section{Besuch von Betreuungseinrichtungen}

In Abbildung 2 wird für die drei- bis siebenjährigen Kinder die periodenspezifische, auf das historische Kalenderjahr bezogene Quote des Besuchs von Betreuungseinrichtungen vor ihrer Einschulung dargestellt. Wie bereits oftmals mit aggregierten Zeitreihen dokumentiert, ist für jedes Jahr im Zeitraum von 1984 bis 2003 die Nutzungsquote bei den Ostdeutschen am höchsten, gefolgt von den Westdeutschen, und die niedrigsten Nutzungsquoten weisen Kinder von Migranten auf (vgl. Kreyenfeld 2004).

5) So ist anzunehmen, dass höher gebildete Eltern oder erwerbstätige Mütter oder Haushalte mit höherem Einkommen ihre Kinder eher in die Einrichtungen vorschulischer Kinderbetreuung schicken als beispielsweise Eltern aus unteren Sozialschichten, die über geringeres Finanz- und Humankapital verfügen. Gerade höher gebildete Eltern kennen die Möglichkeiten und Auswirkungen der frühen vorschulischen Bildung besser als die weniger gebildeten Eltern. Erwerbstätige Mütter sind in der Regel höher gebildet und nutzen eher die Optionen für die Vereinbarkeit von Familie und Beruf, die durch die vorschulische Kinderbetreuung gegeben ist. Diese Eltern haben nicht nur die Ressourcen für die Nutzung von Angeboten der vorschulischer Bildung und Kinderbetreuung, sondern sie verfügen bereits über die Ressourcen, die zu privilegierten Bildungschancen ihrer Kinder führen - und zwar unabhängig davon, ob die Eltern ihre Kinder in die Kindergärten oder auf Vorschulen schicken oder nicht. So bleibt zunächst unklar, ob die besseren Bildungschancen ihrer Kinder kausal auf ihre vorschulische Bildung zurückgeführt werden können oder schlichtweg auf der begünstigenden Sozialisation im Elternhaus beruhen.

6) Das Matching-Verfahren ist angesichts des geringen Stichprobenumfangs für die Schulkinder kaum praktikabel. Wenn zwischen zwei Drittel und drei Viertel der Kinder im Vorschulalter die Kinderbetreuungseinrichtungen besuchen, dann wird es schwierig, für die abstinenten Kinder in ausreichender Zahl einen »Zwilling « zu finden. Diese technische Schwierigkeit wächst mit der Zahl der Variablen, die man für die Paarzusammenstellung verwenden möchte, so dass dann die Analysestichprobe immer geringer ausfällt. 
Abbildung 2: Besuch von vorschulischen Betreuungs- und Bildungseinrichtungen der 3 bis 7-jährigen Kinder vor der Einschulung (Deutschland, 1984-2003)

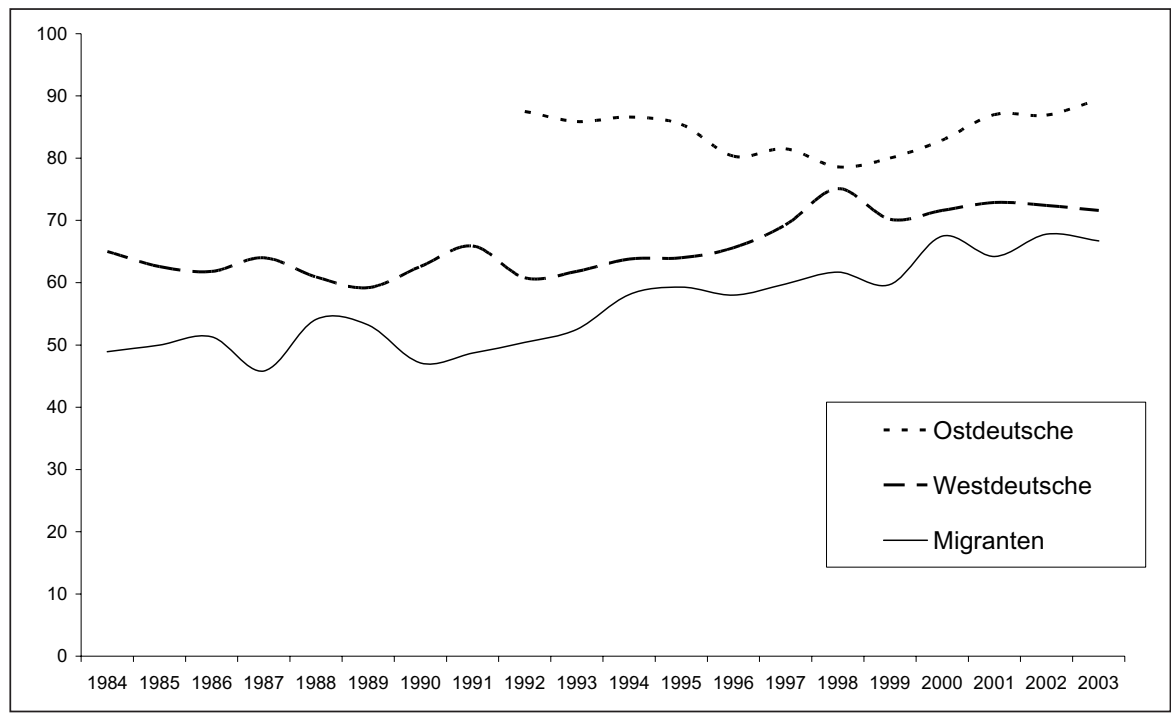

Quelle: SOEP 1984-2003 (ungewichtete Ergebnisse) - eigene Berechnung

So liegen deren Quoten in den einzelnen Jahren zwischen 49 und 67 Prozent. Allenfalls zwei Drittel der Migrantenkinder im Alter zwischen drei und sieben Jahren besuchen die vorschulischen Einrichtungen, insbesondere den Kindergarten, während fast drei Viertel der westdeutschen Kinder und mehr als 80 Prozent der ostdeutschen Kinder in diesem Lebensalter in vorschulischen Einrichtungen betreut werden. Gemessen an der Partizipation ist bei den Deutschen - im Unterschied zu den Kindern mit Migrationsstatus - der Kindergarten zur »Regel-Einrichtung« geworden (vgl. Kreyenfeld et al. 2002).

Der Besuch vorschulischer Bildungseinrichtungen differiert nach dem Alter der Kinder: Mit zunehmendem Alter und dem Näherrücken der Einschulung wird der Besuch von Kindergarten und Vorschule immer wahrscheinlicher (Tabelle 1). Diese Entwicklungen sind für die einzelnen Subpopulationen unterschiedlich. Die meisten Kinder in allen drei Subpopulationen besuchen im Alter von sechs Jahren vorschulische Betreuungseinrichtungen.

Tabelle 1: Besuch einer vorschulischen Betreuungs- und Bildungseinrichtung nach Alter (Abstromprozente)

\begin{tabular}{|c|c|c|c|}
\hline Alter & Westdeutsche & Ausländer & Ostdeutsche \\
\hline & & & \\
3 & 15,9 & 9,2 & 65,7 \\
4 & 57,4 & 44,7 & 85,5 \\
5 & 87,9 & 67,2 & 90,5 \\
6 & 93,2 & 73,7 & 92,1 \\
7 & 87,6 & 72,9 & 91,5 \\
Insgesamt & 65,7 & 50,9 & 84,9 \\
\hline
\end{tabular}

Quelle: SOEP1984-2003 (ungewichtete Ergebnisse) - eigene Berechnung 
Während in Ostdeutschland die meisten Kinder bereits im Alter von drei Jahren in diesen Einrichtungen sind, setzt in Westdeutschland der Besuch erst im vierten oder fünften Lebensjahr ein. Folgende Gründe dürften plausibel sein. In Ostdeutschland führt neben der fortgesetzten Tradition aus DDR-Zeiten das strukturelle Angebot an vorschulischer Kinderbetreuung auch zur entsprechenden Nutzung, die durch die starke Erwerbsorientierung ostdeutscher Mütter gefördert wird. Die soziokulturelle Distanz zu Bildungsinstitutionen in Deutschland ist möglicherweise neben gegebenen Alternativen zur institutionellen Kinderbetreuung im Familienverband der entscheidende Grund für die vergleichsweise niedrigen Besuchsquoten bei den Migranten.

\section{Soziale Selektivität bei der Nutzung von vorschulischen Einrichtungen}

Möchte man die Bildungseffekte vorschulischer Bildung und Betreuung als Kausalität beurteilen, ist es wie bereits ausgeführt notwendig - abgesehen vom strukturellen Angebot an vorschulischer Bildung -, die Sozialstruktur bei der Nutzung von Kindergärten und Vorschulen zu berücksichtigen. Daher schätzen wir mittels logistischer Regression (Long 1997) den Übergang in vorschulische Einrichtungen in Abhängigkeit von sozioökonomischen Ressourcen und Gelegenheiten des Elternhauses. Die empirischen Befunde zeigen zunächst für alle Sozialgruppen, dass in der Abfolge von Kohorten oder im historischen Ablauf die Chancen gestiegen sind, vorschulische Bildungseinrichtungen zu besuchen (Tabelle 2). ${ }^{7}$ Zumindest für ältere Kinder ist es wahrscheinlich, vor ihrer Einschulung den Kindergarten oder die Vorschule besucht zu haben.

7) Der Einfluss erklärender Variablen auf die Wahrscheinlichkeit, vorschulische Einrichtungen im Alter zwischen 3 und 7 Jahren genutzt zu haben, wird mittels logistischer Regression geschätzt (Long 1997). Die Schätzergebnisse werden als so genannte odds ratio dokumentiert (Mare 1980, 1981). Sie bemessen die relative Chance, in Abhängigkeit der erklärenden Variable Kindergarten oder Vorschule zu besuchen. Ein odds-ratio-Wert von größer als 1 weist auf einen positiven Einfluss und ein Wert kleiner als 1 auf einen negativen Einfluss hin. Ist der Wert gleich 1, so gibt es keinen Zusammenhang oder Unterschied nach Merkmalsausprägungen. Der Wert 3,67 im ersten Modell für die Westdeutschen besagt, dass die relative Chance für vorschulische Betreuung mit jedem zusätzlichem Lebensalter um das 3,67-fache ansteigt. Der Wert 3,05 für die Erwerbstätigkeit der Mutter beim Modell für die ostdeutschen Kinder heißt: Kinder erwerbstätiger Mütter haben eine mehr als drei Mal so große Chance für vorschulische Betreuung als diejenigen, deren Mutter nicht erwerbstätig ist. 
Tabelle 2: Determinanten des Besuchs einer vorschulischen Betreuungs- und Bildungseinrichtung - Drei- bis siebenjährige Kinder in der Zeit von 1984 bzw. 1992 bis 2003 (odds ratios - geschätzt mit logistischer Regression)

\begin{tabular}{|c|c|c|c|c|c|c|}
\hline & \begin{tabular}{|l|} 
West- \\
deutsche \\
(Modell
\end{tabular} & $\begin{array}{l}\text { West- } \\
\text { deutsche } \\
\text { (Modell }\end{array}$ & $\begin{array}{l}\text { Migranten } \\
\text { (Modell 1) }\end{array}$ & $\begin{array}{l}\text { Migranten } \\
\text { (Modell 2) }\end{array}$ & $\begin{array}{l}\text { Migranten } \\
\text { (Modell 3) }\end{array}$ & $\begin{array}{l}\text { Ost- } \\
\text { deutsche }\end{array}$ \\
\hline \multicolumn{7}{|l|}{ Zeitdimensionen } \\
\hline Alter & $3,67 * * *$ & $3,61 * * *$ & $2,61 * * *$ & $2,69 * * *$ & $2,49 * * *$ & $1,74 * * *$ \\
\hline Periode & $1,03 * * *$ & $1,03^{* * *}$ & $1,04 * * *$ & $1,03^{* * *}$ & $1,03 * * *$ & 0,97 \\
\hline \multicolumn{7}{|l|}{$\begin{array}{l}\text { Ressourcen des } \\
\text { Haushalts }\end{array}$} \\
\hline Haushaltsgröße & $0,89 * * *$ & $0,93 *$ & $0,80 * * *$ & $0,85 * * *$ & $0,87 * * *$ & $0,62 * * *$ \\
\hline Haushaltseinkommen & $1,06^{* * *}$ & 1,02 & $1,11 * * *$ & $1,06^{*}$ & $1,07 \dagger$ & $1,15 \dagger$ \\
\hline $\begin{array}{l}\text { Bildungsniveau der } \\
\text { Mutter }\end{array}$ & 1,02 & 1,01 & $1,10 * * *$ & $1,06^{*}$ & $1,06 \dagger$ & 1,06 \\
\hline $\begin{array}{l}\text { Bildungsniveau des } \\
\text { Vaters }\end{array}$ & $1,05^{* * *}$ & $1,04 * * *$ & $1,04 *$ & $1,04 \dagger$ & 1,05 & \\
\hline \multicolumn{7}{|l|}{ Berufliche Integration } \\
\hline $\begin{array}{l}\text { Erwerbstätigkeit der } \\
\text { Mutter }\end{array}$ & & $1,39 * * *$ & & $1,78^{* * *}$ & $1,88 * * *$ & $3,05^{* * *}$ \\
\hline $\begin{array}{l}\text { Ref.: Keine Erwerbs- } \\
\text { tätigkeit }\end{array}$ & & 1 & & 1 & 1 & 1 \\
\hline $\begin{array}{l}\text { Sozioökonomischer } \\
\text { Status }\end{array}$ & & $1,01 * * *$ & & $1,01 * * *$ & $1,02 * * *$ & $1,01 *$ \\
\hline \multicolumn{7}{|l|}{ Kulturelle Integration } \\
\hline Mutter & & & & & $1,44 * * *$ & \\
\hline Vater & & & & & 0,90 & \\
\hline \multirow{2}{*}{$\begin{array}{l}\text { Pseudo-R }{ }^{2} \text { (Cox- } \\
\text { Snell) } \\
\text { Pseudo-R }{ }^{2} \\
\text { (Nagelkerke) } \\
\text { Besuch in \% } \\
\text { N (Insgesamt) }\end{array}$} & $\begin{array}{l}0,33 \\
0,46\end{array}$ & $\begin{array}{l}0,33 \\
0,46\end{array}$ & $\begin{array}{l}0,26 \\
0,35\end{array}$ & $\begin{array}{l}0,28 \\
0,38\end{array}$ & $\begin{array}{l}0,27 \\
0,36\end{array}$ & $\begin{array}{l}0,14 \\
0,24\end{array}$ \\
\hline & $\begin{array}{l}62,5 \\
7.927\end{array}$ & $\begin{array}{l}63,1 \\
7.387\end{array}$ & $\begin{array}{l}53,7 \\
4.132\end{array}$ & $\begin{array}{l}54,8 \\
3.632\end{array}$ & $\begin{array}{l}52,8 \\
2.391\end{array}$ & $\begin{array}{l}85,8 \\
2.164\end{array}$ \\
\hline
\end{tabular}

$* \mathrm{p}=0.05 ; * * \mathrm{p}=0.01 ; * * * \mathrm{p}=0.01 ; \dagger \mathrm{p}=0.1$

Quelle: SOEP (ungewichtete Ergebnisse) - eigene Berechnung

Verfügen Haushalte über höhere Einkommen, dann besuchen Kinder vorschulische Einrichtungen eher als Kinder einkommensschwächerer Eltern: Die soziale Selektivität der Nutzung vorschulischer Kinderbetreuung ergibt sich der Humankapitaltheorie zufolge aus der Relation verfügbarer Ressourcen und erwarteter Nutzen (G.S. Becker 1975). Haushalte mit höheren Einkommen investieren nicht nur eher in die allgemeine Ausbildung ihrer Kinder, sondern auch eher in die gebührenpflichtige vorschulische Kinderbetreuung, weil anfallende Investitions- und Opportunitätskosten geringer sind als der erwartete Nutzen.

Je größer der Haushalt ist, desto seltener besuchen Kinder vorschulische Einrichtungen. Je größer ein Haushalt ist, umso eher sind jedoch Alternativen für eine Betreuung jüngerer Kinder innerhalb des Haushaltes vorhanden oder desto größer sind auch bei einer höheren Kinderzahl 
die anfallenden Kosten für eine außerhäusliche Kinderbetreuung. Von der alternativen Möglichkeit für eine kostengünstige Kinderbetreuung machen vor allem Migranten Gebrauch. In diesem Fall ist es wahrscheinlich, dass Investitionen in die vorschulische Bildung ausbleiben.

Je höher das Bildungsniveau der Eltern ist, desto eher besuchen Kinder vorschulische Bildungseinrichtungen; dies ist vor allem wiederum bei den Migranten der Fall, während bei den Ostdeutschen keine Bildungsselektivitäten vorliegen. Eltern mit höherem Bildungsniveau sind nicht nur eher mit dem Bildungssystem und dem Wert von frühen und stetigen $\mathrm{Hu}-$ mankapitalinvestitionen vertraut, sondern auch eher in der Lage, Bildungsrenditen langfristig zu diskontieren (Hillmert und Jacob 2003). Dies gilt insbesondere für erwerbstätige Mütter, die in der Regel ein höheres Bildungsniveau aufzeigen, und für beruflich erfolgreiche Haushalte, die den höheren Sozialschichten angehören und am Bildungserfolg ihrer Kinder besonders interessiert sind. Diese Familien sind daher eher bereit, in die vorschulische Bildung ihrer Kinder zu investieren.

Je höher der sozioökonomische Status des Elternhauses ist, desto eher erfolgt eine außerhäusliche Kinderbetreuung in vorschulischen Einrichtungen. Da bei Kontrolle des sozioökonomischen Status der Einfluss des Haushaltseinkommens auf die Nutzung vorschulischer Einrichtungen nur noch auf dem 10-Prozent-Niveau signifikant ist, kann davon ausgegangen werden, dass damit hauptsächlich die Verfügbarkeit über Finanzmittel indiziert wird, die für frühe Bildungsinvestitionen mobilisiert werden können.

Je besser die soziale Integration des Elternhauses durch die Erwerbstätigkeit der Mutter gelingt, desto eher partizipieren Kinder auch an vorschulischer Betreuung. Gerade die vorschulische Bildung der Kinder bietet den erwerbstätigen Müttern die Möglichkeit, auf einem kostengünstigen Wege die Renditen ihrer eigenen Humankapitalinvestitionen langfristig zu sichern (Kreyenfeld et al. 2002, S. 205-206; Roßbach 2000, S. 27). Voraussetzung dafür ist die erfolgreiche Vereinbarkeit von Familie und Beruf, die durch entsprechende Angebote an außerfamiliärer institutioneller Kinderbetreuung vereinfacht wird und den erfreulichen $\mathrm{Ne}$ beneffekt hat, dass Opportunitätskosten wegen Reduzierung oder Aufgabe einer Erwerbstätigkeit vermieden werden können (Spieß und Tietze 2002, S. 143-144). Des Weiteren ist vorschulische Bildung eine rationale Strategie, negative Konsequenzen eines misslungenen intergenerationalen Transfers von Humankapital wegen geringer Humankapitalinvestitionen von Migranten zu kompensieren: Je höher die Nachfrage nach dem Humankapital und -vermögen des Kindes und je höher die Investitionskosten für die Kinder sind, desto notwendiger und wertvoller ist die Erwerbstätigkeit der Mutter, damit die Eltern zusätzliche Einkommen für die Bildung und Ausbildung der Kinder erwirtschaften. Wenn Mütter aufgrund des institutionellen Angebots an Kinderbetreuung auch Familie und Beruf miteinander vereinbaren können, dann sinken die relativen Investitionskosten für die Ausbildung der Kinder (Chiswick 1988), während gleichzeitig die Eltern-Kind-Beziehung an Qualität gewinnt, die Sozialintegration des Elternhauses über den Arbeitsmarkt und die sozialen Beziehungen im Erwerbsleben zunehmen (Roßbach 2000). Die strukturelle Notwendigkeit der externen Kinderbetreuung, um die Vereinbarkeit von Familie und Beruf zu sichern, verliert an Trivialität, wenn berücksichtigt wird, dass dieser Zusammenhang vor allem bei Migranten auftritt, die die besten Alternativen zur institutionellen außerhäuslichen Betreuung aufweisen.

Schließlich ist bei Migranten der Besuch vorschulischer Bildung am wahrscheinlichsten, wenn die Mutter über gute (selbst eingeschätzte) Fähigkeiten verfügt, Deutsch zu sprechen. Neben Unkenntnis von Struktur und institutionellen Regelungen des deutschen Bildungssystems sind sprachliche Probleme entscheidende Hindernisse für die Übergabe der Kinder in die vorschulischen Einrichtungen. Somit wäre zu erwarten, dass höher gebildete ausländische Eltern, die in der Regel auch eher der deutschen Sprache mächtig sind, die Möglichkeiten der vorschulischen Kinderbetreuung nutzen. Aus deren Sicht wären frühe Investitionen in das Humankapital ihrer Kinder eine Strategie für soziale Integration und gesellschaftliche 
Teilhabe im Ankunftsland (Nauck et al. 1998; Diefenbach und Nauck 1997). Diese Annahmen werden durch unsere Daten unterstützt.

Insgesamt belegen unsere Befunde, dass berufliche Integration, kulturelle Assimilation und verfügbares Human- und Kulturkapital die wichtigsten Mechanismen darstellen, dass Kinder mit Migrationshintergrund in Deutschland vorschulische Betreuungseinrichtungen besuchen. Für einheimische Kinder hängen Partizipationschancen vornehmlich von vorteilhaften sozioökonomischen Ressourcen im Elternhaus ab.

Bei den Migranten könnte man noch zusätzlich argumentieren, dass nicht alleine der Migrantenstatus, sondern die Zugehörigkeit zu einer bestimmten Nationalität ausschlaggebend dafür ist, ob die Kinder vorschulische Betreuungseinrichtungen besuchen oder nicht. Oder anders ausgedrückt: Es könnte auch Unterschiede zwischen den einzelnen Nationalitäten geben und dass es daher die im vorschulischen und schulischen Bildungssystem benachteiligten Kinder von Migranten nicht gibt. Daher wird für die Bestimmungsgründe des Besuchs vorschulischer Betreuungs- und Bildungseinrichtungen auch die Nationalität der Kinder berücksichtigt.

Tabelle 3: Determinanten des Besuchs einer vorschulischen Bildungseinrichtung - nur dreibis siebenjährige Kinder von Migranten (odds ratio - geschätzt mit logistischer Regression)

\begin{tabular}{|l|lll|}
\hline & Modell 1 & Modell 2 & Modell 3 \\
\hline Sozialer Hintergrund & & & \\
Alter & $2,55^{* * *}$ & $2,70^{* * *}$ & $2,52^{* * *}$ \\
Periode & $1,06^{* * *}$ & $1,04^{* * *}$ & $1,02^{* * *}$ \\
Haushaltsgröße & & $0,87^{* * *}$ & $0,89^{* * *}$ \\
Haushaltseinkommen & & $1,06^{*}$ & $1,07^{*}$ \\
Bildungsniveau der Mutter & & $1,06^{*}$ & $1,06 \dagger$ \\
Bildungsniveau des Vaters & & $1,05^{*}$ & $1,06 \dagger$ \\
Erwerbstätigkeit der Mutter & & $1,79^{* * *}$ & $1,93^{* * *}$ \\
Ref.: Keine Erwerbstätigkeit der Mutter & & 1 & 1 \\
Sozioökonomischer Status & & $1,01^{* * *}$ & $1,01^{*}$ \\
Deutsch sprechen (Mutter) & & & $1,46^{* * *}$ \\
Deutsch sprechen (Vater) & & & 0,97 \\
Nationalität des Haushaltsvorstandes & & & \\
türkisch & & 0,67 & 0,94 \\
(ex-)jugoslawisch & $0,54^{*}$ & 0,65 & 0,59 \\
griechisch & 0,68 & 0,65 & 0,85 \\
italienisch & 0,75 & 0,88 & 1,17 \\
deutsch & 0,80 & 0,63 & 1,33 \\
Ref.: Andere Nationalitäten & 0,87 & 1 & 1 \\
\hline Pseudo-R ${ }^{2}$ (Cox-Snell) & 1 & 0,28 & 0,27 \\
Pseudo- ${ }^{2}$ (Nagelkerke) & 0,25 & 0,38 & 0,37 \\
Besuch in \% & 0,34 & 3.632 & 2,391 \\
N (Insgesamt) & 53,8 & & \\
\hline
\end{tabular}

$* \mathrm{p}=0.05 ; * * \mathrm{p}=0.01 ; * * * \mathrm{p}=0.01 ; \dagger \mathrm{p}=0.1$

Quelle: SOEP (ungewichtete Ergebnisse) - eigene Berechnung 
Im ersten sehr einfachen Schätzmodell deutet es sich an, dass gerade türkische Kinder im Vergleich zu anderen Nationalitäten die vergleichsweise geringsten Chancen haben, an der vorschulischen Bildung zu partizipieren (Tabelle 3). Allerdings verschwinden die nationalen Unterschiede gänzlich, wenn Ressourcen des Elternhauses kontrolliert werden. Es ist eben nicht die nationale Herkunft oder Zugehörigkeit zu einer bestimmten Nationalität, die die frühen Bildungschancen determinieren, sondern offenkundig - wie von der Humankapitaltheorie prognostiziert - Ressourcen, die für die Ausbildung der Kinder mobilisiert werden können.

\section{Bildungseffekte vorschulischer Betreuung}

Die ungleiche Partizipation an früher Elementarbildung findet ihre Fortsetzung in den weiterführenden Schulen in der Sekundarstufe I. Mehr als 10 Prozent der ostdeutschen und rund ein Viertel der westdeutschen Jugendlichen, für die zuvor die Beteiligung an vorschulischer Betreuung betrachtet wurde, besuchen - betrachtet für den Zeitraum von 1992 bis 2003 - im Alter von 14 Jahren die Hauptschule. Hingegen wechselt zwischen einem und zwei Drittel der Migranten auf die Hauptschule, wobei Abwanderungen aus Deutschland unberücksichtigt bleiben. Insofern haben sich relative Bildungserfolge für diese Jugendlichen ergeben, die zu einem wachsenden Anteil auf die Real- oder Gesamtschulen sowie auf das Gymnasium gewechselt sind. Allerdings haben im Jahre 2003 Migranten immerhin noch ein 3,6 Mal höheres Risiko als Ostdeutsche und ein 1,7 Mal höheres Risiko als Westdeutsche, nach der Grundschule auf die Hauptschule zu wechseln.

Tabelle 4: Bildungsbeteiligung von 14-jährigen nach Besuch vorschulischer Bildungseinrichtungen (Abstromprozente für 1992-2003)

\begin{tabular}{|c|c|c|c|c|c|c|c|c|c|}
\hline \multirow[t]{2}{*}{$\begin{array}{l}\text { Besuch vorschulischer } \\
\text { Bildungseinrichtung }\end{array}$} & \multicolumn{3}{|c|}{ Westdeutsche } & \multicolumn{3}{|c|}{ Migranten } & \multicolumn{3}{|c|}{ Ostdeutsche } \\
\hline & HS & RS & GYM & HS & RS & GYM & HS & RS & GYM \\
\hline $\mathrm{Ja}$ & 27,0 & 31,0 & 42,0 & 49,0 & 29,4 & 21,6 & 11,9 & 46,4 & 41,8 \\
\hline Nein & 50,8 & 23,7 & 25,4 & 58,9 & 33,7 & 7,4 & 5,9 & 70,6 & 23,5 \\
\hline Insgesamt & 28,6 & 30,5 & 41,0 & 51,7 & 30,6 & 17,7 & 11,4 & 48,3 & 40,3 \\
\hline Cramér's V & & 0,13 & & & 0,17 & & & 0,13 & \\
\hline
\end{tabular}

Quelle: SOEP (ungewichtete Ergebnisse) - eigene Berechnung

Ungeachtet der sozialen Selektivität bei der Nutzung vorschulischer Einrichtungen, können wir anhand einfacher Deskriptionen den Einfluss von vorschulischer Bildung auf die späteren Bildungschancen - gemessen anhand der Verteilung auf die stratifizierten Schullaufbahnen in der Sekundarstufe I - abschätzen (Tabelle 4). Zunächst werden sowohl bei den Einheimischen als auch bei den Migranten die Bildungschancen gefördert, wenn sie vor der Einschulung Kindergarten oder Vorschule besuchen. Während rund 27 Prozent der westdeutschen Schulkinder, die eine vorschulische Einrichtung besucht haben, am Ende ihrer Grundschulzeit auf die unterste Schullaufbahn, die Hauptschule, rund 31 Prozent in die Realschule, die mittlere Schullaufbahn, und schließlich 42 Prozent auf das Gymnasium, der höchsten Schullaufbahn, wechseln, wechselt hingegen die Hälfte der westdeutschen Schulkinder ohne Erfahrung mit vorschulischer Bildung in die Hauptschule über und rund ein 
Viertel auf das Gymnasium. Ähnliche Verteilungen ergeben sich für die ostdeutschen Schulkinder. Bei den Migrantenkindern besucht nach dem Bildungsübergang mehr als die Hälfte der 14-Jährigen die Hauptschule, ein Drittel die Realschule und weniger als ein Zehntel das Gymnasium, wenn sie keine vorschulischen Einrichtungen besucht haben. Hingegen wechselt fast ein Viertel der Migrantenkinder auf das Gymnasium und weniger als die Hälfte auf die Hauptschule, wenn sie Erfahrungen mit Kindergarten und Vorschule haben.

Trotz alledem sollten für die Kinder von Migranten die Bildungseffekte vorschulischer Bildung nicht überschätzt werden, wie folgender Vergleich mit den relativen Bildungschancen für einheimische Jugendliche zeigt: Westdeutsche Schulkinder mit Kindergarten- und Vorschulbesuch haben eine doppelt so große Chance, auf das Gymnasium zu wechseln, als diejenigen ohne jegliche vorschulische Bildung. Ähnliche Chancenstrukturen bestehen bei ostdeutschen Schulkindern, wobei diejenigen ohne vorschulische Bildung bessere Chancen haben, mindestens auf die Realschule zu wechseln, als dies bei den Westdeutschen der Fall ist. Ohne Kindergarten- und Vorschulbesuch haben westdeutsche Schulkinder ein 2,7-Mal größeres Risiko, in die Hauptschule zu gelangen, als Westdeutsche mit Kindergarten- und Vorschulbesuch.

Hingegen haben Kinder von Migranten - unabhängig davon, ob sie Kindergärten bzw. Vorschulen besucht haben oder nicht - deutlich ungünstigere Bildungschancen als Einheimische. So haben Autochthone rund 3-Mal bessere Chancen, auf das Gymnasium zu wechseln, während Migrantenkinder ein fast 9-Mal höheres Risiko haben, in die Hauptschule zu wechseln. Besuchen sie vorschulische Einrichtungen, dann haben sie zwar 3,4-Mal bessere Chancen, auf das Gymnasium zu wechseln, als ihre Landsleute ohne jegliche Erfahrung mit Kindergarten und Vorschule. Verglichen mit einheimischen Schulkindern, die ebenfalls vorschulische Betreuungseinrichtungen besucht haben, sind die Migranten trotz dieser Startvorteile immer noch deutlich im Nachteil beim Übergang in die Sekundarstufe I: Einheimische mit vorschulischer Bildung haben immer noch 2,5-Mal bessere Chancen auf das Gymnasium zu wechseln, während Migrantenkinder mit vorschulischer Bildung die gleichen Bildungschancen haben wie Einheimische ohne vorschulische Bildung.

Sicherlich gibt es keine homogenen Bildungschancen für die betrachteten Populationen, wenn zusätzlich weitere Ressourcen des Elternhauses in Betracht gezogen werden. Offensichtlich bestimmen sozioökonomische Ressourcen des Elternhauses und das kulturelle Kapital der Eltern die Bildungschancen von Kindern. Werden diese Ressourcen berücksichtigt, dann gibt es nur noch für die Migranten einen signifikanten Bildungseffekt vorschulischer Betreuung (Tabelle 5). 
Tabelle 5: Einfluss des Besuchs vorschulischer Kinderbetreuungseinrichtungen auf die Bildungsbeteiligung von 14-jährigen Schulkindern, 1992-2003 (odds ratios - geschätzt mit multinomialer bzw. binärer Logit-Regression bei Kontrolle sozialer Selektivität des Besuchs vorschulischer Bildungseinrichtungen; in eckigen Klammern: Kehrwert des odds ratio)

\begin{tabular}{|c|c|c|c|c|c|}
\hline & \multicolumn{2}{|c|}{ Westdeutsche } & \multicolumn{2}{|c|}{ Migranten } & \multirow{2}{*}{$\begin{array}{l}\text { Ostdeutsche } \\
\text { Gymna- } \\
\text { sium }\end{array}$} \\
\hline & $\begin{array}{l}\text { Real- } \\
\text { schule }\end{array}$ & $\begin{array}{l}\text { Gymna- } \\
\text { sium }\end{array}$ & $\begin{array}{l}\text { Real- } \\
\text { schule }\end{array}$ & $\begin{array}{l}\text { Gymna- } \\
\text { sium }\end{array}$ & \\
\hline$\lambda_{\text {(vorschulische Bildung) }}$ & 1,33 & 1,18 & 0,95 & $1,82 *$ & 0,80 \\
\hline Junge & 0,77 & $\begin{array}{c}0,65^{*} \\
{[1 / 1,54]}\end{array}$ & 0,71 & 1,09 & $\begin{array}{c}0,52^{*} \\
{[1 / 1,91]}\end{array}$ \\
\hline Mädchen & 1 & 1 & 1 & 1 & 1 \\
\hline $\begin{array}{l}\text { Bildungsniveau der } \\
\text { Mutter }\end{array}$ & $1,19 * *$ & $1,50 * * *$ & $1,08^{*}$ & $1,21 * *$ & $1,15^{*}$ \\
\hline Sozioökonomischer Status & $1,03^{* * *}$ & $1,06^{* * *}$ & 1,01 & $1,03^{*}$ & $1,05^{*}$ \\
\hline Periode & 1,02 & 0,98 & $1,72 *$ & 1,66 & 1,25 \\
\hline $\begin{array}{l}\text { Pseudo- } \mathrm{R}^{2} \\
\mathrm{~N}\end{array}$ & & & & & $\begin{array}{c}0,133 \\
188\end{array}$ \\
\hline
\end{tabular}

$* \mathrm{p}=0.05 ; * * \mathrm{p}=0.01 ; * * * \mathrm{p}=0.01 ; \dagger \mathrm{p}=0.1$

Quelle: SOEP (ungewichtete Ergebnisse) - eigene Berechnung

Bei diesem Bildungseffekt ist die soziale Selektivität bei der Partizipation an vorschulischer Betreuung und Bildung in Form von bedingten Wahrscheinlichkeiten, diese Einrichtungen besucht zu haben, berücksichtigt. Die Kontrolle der sozialen Selektivität bei der Nutzung vorschulischer Kinderbetreuung basiert auf den in Tabelle 2 dokumentierten Schätzungen. So haben Migranten, die vor ihrer Einschulung Kindergarten und Vorschule besucht haben, signifikant bessere Chancen, auf das Gymnasium zu wechseln, als ihre Landsleute ohne vorschulische Bildung. So gesehen, rentieren sich für Migranten entsprechende Investitionen in vorschulische Bildung, auch wenn dadurch ihre relativen Nachteile beim Bildungserwerb gegenüber den einheimischen Schulkindern nicht wettgemacht werden können. Hiermit bestätigen wir die Befunde von Büchel et al. (1997). ${ }^{8}$

8) Die Abweichung von früheren Ergebnissen mit den gleichen Daten hat zwei Gründe: Erstens werden im Unterschied zu einer früheren Analyse nicht nur die letzte, sondern nunmehr alle Partizipationen vor der Einschulung berücksichtigt (vgl. Becker und Lauterbach 2004). Dadurch wird bei den Einheimischen die Kontrollgruppe der Nichtteilnehmer sehr klein und daher sind Bildungseffekte kaum noch zu identifizieren. Zweitens vermuten wir, dass die Ergebnisse zu den Bildungseffekten periodenspezifisch sind. Da nunmehr ein längerer Zeitraum beobachtet wird, kann es zu Schwankungen in den Signifikanzen kommen. 
Tabelle 6: Bildungsbeteiligung von 14-jährigen Schulkindern mit Migrationshintergrund, 1992-2003 (odds ratio - geschätzt mit multinomialer Logit-Regression; in eckigen Klammern: Kehrwert des odds ratio)

\begin{tabular}{|c|c|c|c|c|c|c|}
\hline & \multicolumn{2}{|c|}{ Modell 1} & \multicolumn{2}{|c|}{ Modell 2} & \multicolumn{2}{|c|}{ Modell 3} \\
\hline & $\begin{array}{l}\text { Real- } \\
\text { schule }\end{array}$ & $\begin{array}{l}\text { Gymna- } \\
\text { sium }\end{array}$ & $\begin{array}{l}\text { Real- } \\
\text { schule }\end{array}$ & $\begin{array}{c}\text { Gymna- } \\
\text { sium }\end{array}$ & $\begin{array}{l}\text { Real- } \\
\text { schule }\end{array}$ & $\begin{array}{c}\text { Gymna- } \\
\text { sium }\end{array}$ \\
\hline$\lambda_{\text {(vorschulische Bildung) }}$ & & & 1,02 & $1,90 * *$ & 0,97 & $1,99 * *$ \\
\hline Junge & & & & & 0,67 & 1,04 \\
\hline Mädchen & & & & & 1 & 1 \\
\hline Periode & & & & & $1,74 *$ & 1,72 \\
\hline Soziale Herkunft & & & & & & \\
\hline $\begin{array}{l}\text { Bildungsniveau der } \\
\text { Mutter }\end{array}$ & & & & & $\begin{array}{c}0,94 \dagger \\
{[1 / 1,07]}\end{array}$ & $1,23 * *$ \\
\hline $\begin{array}{l}\text { Sozioökonomischer } \\
\text { Status }\end{array}$ & & & & & 0,99 & $1,03 *$ \\
\hline Nationale Herkunft & & & & & & \\
\hline Türkei & 0,73 & $\begin{array}{c}0,63 \dagger \\
{[1 / 1,59]}\end{array}$ & 0,73 & 0,67 & 1,38 & 0,82 \\
\hline Jugoslawien & 0,75 & 0,70 & 0,75 & 0,72 & 0,84 & 0,85 \\
\hline Griechenland & $2,18^{*}$ & $3,47 * *$ & $2,17^{*}$ & $3,81 * *$ & $2,37^{*}$ & $3,95 * *$ \\
\hline Italien & $\begin{array}{c}0,58^{*} \\
{[1 / 1,72]}\end{array}$ & $\begin{array}{c}0,48^{*} \\
{[1 / 2,07]}\end{array}$ & $\begin{array}{c}0,48^{*} \\
{[1 / 2,09]}\end{array}$ & $\begin{array}{c}0,45^{*} \\
{[1 / 2,22]}\end{array}$ & $\begin{array}{c}0,58^{*} \\
{[1 / 1,72]}\end{array}$ & $\begin{array}{c}0,43 * \\
{[1 / 2,32]}\end{array}$ \\
\hline Spanien & 1,52 & 1,25 & 1,53 & 1,11 & 1,18 & 0,50 \\
\hline Andere Nationalität & 1 & 1 & 1 & 1 & 1 & 1 \\
\hline $\begin{array}{c}\text { Pseudo- } \mathrm{R}^{2} \\
\mathrm{~N}\end{array}$ & & & & & & \\
\hline
\end{tabular}

$* \mathrm{p}=0.05 ; * * \mathrm{p}=0.01 ; * * * \mathrm{p}=0.01 ; \dagger \mathrm{p}=0.1$

Quelle: SOEP (ungewichtete Ergebnisse) - eigene Berechnung

In Bezug auf die Ungleichheit von Bildungschancen bei den Migranten gibt es eine Binnendifferenzierung zwischen den Nationalitäten (Alba et al. 1994; Esser 2001). In Deutschland haben Griechen deutlich günstigere Bildungschancen als die größte Ausländergruppe in Deutschland, die Türken. Die Italiener, die am längsten in Deutschland leben, weisen noch schlechtere Bildungserfolge als die Türken auf. Dies spricht gegen die These eines kulturellen Defizits an in der Schule nachgefragten Kenntnissen und Verhaltensweisen bei den Migranten. Diese vorliegenden Befunde, die unter anderem mit demselben Datensatz generiert wurden, können wir auch dann bestätigen, wenn die Partizipation an vorschulischen Bildungseinrichtungen kontrolliert wird (Tabelle 6). So haben Griechen die günstigsten Bildungschancen unter den Migranten, während die Italiener die ungünstigsten Bildungschancen aufweisen. Die relativen Nachteile von türkischen Schulkindern beim Übergang in das Gymnasium erweist sich als zufällig, wenn der Besuch vorschulischer Einrichtungen in Rechnung gestellt wird.

Die Binnendifferenzierung von Bildungschancen bei den Migranten ist auch dann noch festzustellen, wenn man die sozioökonomischen Ressourcen und das Bildungskapital der El- 
tern berücksichtigt. Offensichtlich spielen auch soziale Bedingungen innerhalb der Gruppe von Migranten eine gewichtige Rolle, die mit dem Migrationsstatus verbunden sind. Als aussagekräftig haben sich in vorliegenden Studien Faktoren wie das Alter der Kinder bei Einwanderung, Dauer des Verbleibs im Ankunftsland, Transferierbarkeit elterlicher Ressourcen für den deutschen Arbeitsmarkt, etc. erwiesen (Steinbach und Nauck 2004).

\section{Schluss}

Das Bildungssystem hat durch die Vermittlung von Wissen und die Vergabe von Zertifikaten einen entscheidenden Einfluss auf die soziale Platzierung seiner Absolventen und daran geknüpfte Lebenschancen: Je höher der erreichte Abschluss, desto größer die Chancen auf den gewünschten Ausbildungs- oder Studienplatz und damit im Allgemeinen auch auf die spätere berufliche Position. Mit der Wahl von Schul- und Ausbildungswegen sind klare Unterschiede im Hinblick auf Einkommenschancen, Aufstiegsmöglichkeiten und Beschäftigungssicherheit im weiteren Berufsleben verbunden. Diese Chancen werden den Migranten im deutschen Bildungssystem in einem geringeren Maße gewährt als den Einheimischen (Diefenbach 2004). Diese Tatsache wiegt aus bildungs- und gesellschaftspolitischer Sicht vor allem deswegen schwer, weil Bildung eine wichtige Voraussetzung für die soziale Integration von Migranten auf annähernd allen relevanten Dimensionen des Lebens in Deutschland darstellt. Nicht zuletzt ist Bildung eine relevante Ressource, bei der ein kleiner Niveauunterschied am Anfang des Bildungsverlaufs zu kumulativen Nachteilen im weiteren Lebensverlauf und im Aggregat zu dauerhaften Ungleichheiten führt. Daher war es Ziel der vorliegenden Untersuchung, die Frage zu klären, ob sich die vorschulische Betreuung, Erziehung und Bildung in Krippenplätzen, Kindergärten, Kindertagesstätten und Vorschulen förderlich auf die Bildungschancen von Jugendlichen auswirken und damit ein sinnvolles Instrumentarium ist, die Nachteile von Migranten im deutschen Bildungssystem zu reduzieren.

Die an die Untersuchungen von Büchel et al. (1997) sowie von Becker und Lauterbach (2004) anknüpfenden empirischen Auswertungen mit den SOEP-Daten belegen positive Bildungseffekte für Migrantenkinder. Die Detailergebnisse sind allerdings ernüchternd. Eine optimistische Sichtweise würde hervorheben, dass gerade die im deutschen Bildungssystem besonders im Nachteil befindlichen Migrantenkinder durch vorschulische Bildung ihre Bildungsaussichten deutlich verbessern können und gegenüber den Migrantenkindern ohne vorschulische Bildung im Vorteil sind. Multivariate Analysen haben bei besonderer Kontrolle des sozial selektiven Besuchs von Kindergarten oder Vorschule sowie der sozialen Herkunft und Nationalität für den Zeitraum von 1992 bis 2003 ergeben, dass Migranten mit Erfahrungen in vorschulischer Bildung eine rund $2 \mathrm{Mal}$ höhere Chance haben, auf das Gymnasium zu wechseln als die altersgleichen Migranten ohne vorschulische Betreuung.

Eine pessimistische Sichtweise ergibt sich dadurch, dass trotz alledem die Bildungsdefizite gegenüber den Einheimischen nur in beschränktem Maße kompensiert werden. So realisieren Migrantenkinder mit vorschulischer Bildung solche Bildungschancen wie deutsche Schulkinder ohne Besuch vorschulischer Kinderbetreuung oder wie deutsche Arbeiterkinder. Durch vorschulische Bildung und Betreuung können die Migrantenkinder zwar ihre Bildungsrückstände gegenüber einheimischen Schulkindern verringern, aber in ihren Bildungschancen nicht zu den Einheimischen aufschließen.

Bei den Einheimischen insgesamt verschiebt sich durch vorschulische Bildung die Trennlinie beim Übergang in die weiterführenden Schullaufbahnen in Richtung Gymnasium vs. den Rest und bei den Migrantenkindern in Richtung Hauptschule vs. den Rest. Optimistisch stimmt wiederum, dass den Migranten dadurch Schlechterstellungen, die mit dem Besuch einer Hauptschule einhergehen, erheblich reduziert werden können, während die Einheimischen ihre ohnehin vergleichsweise günstigeren Bildungschancen noch weiter verbessern 
können. Andererseits haben wir damit einen weiteren Grund für die Kumulation sozial benachteiligter Schulkinder in der Hauptschule aufgedeckt (Solga und Wagner 2001). Es sind neben den Kindern aus unteren Sozialschichten diejenigen mit Migrationshintergrund, die nicht die vorschulischen Kinderbetreuungseinrichtungen besuchen (konnten), die dann die ungünstigen Konsequenzen des Hauptschulbesuches tragen müssen. Allerdings ist zu berücksichtigen, dass mit den herangezogenen Daten die Wirkungen vorschulischer Bildung auf die Chancen bei der Einschulung und auf die schulischen Leistungen in der Grundschulzeit nicht untersucht werden können, so dass relevante Selektionsmechanismen im Bildungssystem unberücksichtigt bleiben.

In theoretischer Hinsicht haben die von uns vorgelegten Ergebnisse eine weitere Bestärkung für die strukturell-individualistische Erklärung der Bildungsnachteile von Migranten im deutschen Bildungssystem geliefert. Zum einen liefern unsere Befunde empirisch abgesicherte Argumente gegen Erklärungsmuster, die ausschließlich auf kulturelle Defizite von Migranten abstellen. Eher ist davon auszugehen, dass Unterschiede in elterlichen Bildungsvorstellungen und -entscheidungen, die durchaus mit Motiven und Konditionen der Einwanderung zusammenhängen, sowie in den sozioökonomisch gegebenen Möglichkeiten, langfristig in die Bildung von Kindern zu investieren, mit Nachteilen von Migranten im deutschen Bildungssystem einhergehen. Deutlicher als der Bildungsverlauf in der Sekundarstufe ist die Partizipation an vorschulischer Betreuung, Erziehung und Bildung dadurch gekennzeichnet. Denn bei Kontrolle der sozioökonomischen Ressourcen des Elternhauses lassen sich keine signifikanten Unterschiede mehr zwischen den Nationalitäten finden. Dass aber die Ressourcenausstattung des Elternhauses die Bildungsmöglichkeiten mitbestimmt, konnte daran abgelesen werden, dass bei Kontrolle des Besuchs vorschulischer Einrichtungen die sozioökonomischen Ressourcen des Elternhauses bedeutsam für die Bildungschancen in der Sekundarstufe sind. Worauf diese letztlich zurückzuführen sind - Mechanismen hierfür wären etwa Lebensplanung der Eltern und darauf aufbauende elterliche Bildungsentscheidungen -, konnte mit den von uns herangezogenen Daten des SOEP nicht untersucht wurden.

Trotz alledem sind die von uns vorgelegten Befunde mit gewissen Einschränkungen zu interpretieren, die sich aus Messungenauigkeiten und fehlenden Informationen ergeben: Erstens führt möglicherweise die durch das SOEP vorgegebene unzureichende Abgrenzung der unterschiedlichen Formen der vorschulischen Kinderbetreuung zu diesem unerwarteten Ergebnis. So ist eine präzise Extraktion von Tagesmüttern aus der heterogenen Kategorie vorschulischer Betreuung erst seit der Befragungswelle im Jahre 2000 möglich.

Zweitens könnten wir es mit einem statistischen Artefakt zu tun haben, weil mangels verfügbarer Informationen im SOEP nicht nur die schulische Leistung der Kinder, sondern auch die Qualität der vor der Einschulung besuchten Betreuungseinrichtungen nicht berücksichtigt werden konnte. Daher müssten in Zukunft der Bildungsverlauf der Kinder und die Auswirkungen der Qualität von Kindergärten auf die schulische Leistung systematisch untersucht werden (vgl. Weißhuhn 2001, S. 11).

Drittens sollte angesichts vorgelegter Befunde der positive Einfluss von Kindergärten oder Vorschule auf Bildungschancen von Migranten nicht überschätzt werden, weil die Bildungseffekte sich auf die Bildungschancen unter den Migranten selbst beschränken und die Bildungsungleichheiten innerhalb dieser Population modifizieren. Im Vergleich zu den Einheimischen erfolgt zwar eine relative Verbesserung, aber keine Aufhebung eklatanter Bildungsdisparitäten, da die Teilnehmer unter den Migrantenkindern allenfalls auf das Niveau der einheimischen Schulkinder angehoben werden, die keine vorschulische Einrichtungen besuchen und daher im Vergleich zu den einheimischen Teilnehmern geringere Bildungschancen haben. 
Viertens gilt es im Hinblick auf eine kausale Interpretation der Bildungseffekte zu berücksichtigen, dass wir keine Informationen über die Bildungsprozesse im Elternhaus und in der Schule während der Grundschulzeit verfügen. So ist es nicht ausgeschlossen, dass wir der vorschulischen Betreuung, Erziehung und Bildung signifikante Bildungseffekte zuschreiben, die sich aus ganz anderen, aber mit den SOEP-Daten nicht beobachteten Quellen ergeben haben.

Und schließlich müssen wir uns fünftens vor Augen halten, dass wir es mit einer hochgradig selektiven Stichprobe zu tun haben, die vor allem die positiven Bildungseffekte bei den Migranten betrifft. Die Evaluation wurde für die Kinder und ihre Eltern vorgenommen, die mindestens acht Jahre am Sozioökonomischen Panel teilgenommen haben. Panelmortalität haben wir nicht berücksichtigt. Ebenso wenig berücksichtigt haben wir, ob die Migrantenkinder in ihrem Heimatland entsprechende Erfahrungen mit vorschulischer und schulischer Bildung gesammelt haben.

\section{Literatur}

Alba, Richard / Handl, Johann / Müller, Walter (1994): Ethnische Ungleichheit im deutschen Bildungssystem, in: Kölner Zeitschrift für Soziologie und Sozialpsychologie 46, S. 209-238.

Arbeitsstab »Forum Bildung«, 2001: Empfehlungen des Forum Bildung. (http://www.bmbf.de/pub/ ermpfehlungen_des_forum_bildung.pdf, Stand: August 2003).

Baumert, Jürgen / Schümer, Gundel (2001): Familiäre Lebensverhältnisse, Bildungsbeteiligung und Kompetenzerwerb, in: Deutsches PISA-Konsortium (Hrsg.), PISA 2000. Basiskompetenzen von Schülerinnen und Schülern im internationalen Vergleich. Opladen: Leske+Budrich, S. 323-407.

Becker, Gary S. (1975): Human Capital: A Theoretical and Empirical Analysis with Special Reference to Education. New York/London: Columbia University Press.

Becker, Rolf / Lauterbach, Wolfgang (2004): Vom Nutzen vorschulischer Kinderbetreuung für Bildungschancen, in: Rolf Becker / Wolfgang Lauterbach (Hrsg.), Bildung als Privileg? Erklärungen und empirische Befunde zu den Ursachen von Bildungsungleichheiten, Wiesbaden: VS-Verlag für Sozialwissenschaften, S. 127-159.

Becker, Rolf (2000): Klassenlage und Bildungsentscheidungen. Eine empirische Anwendung der WertErwartungstheorie, in: Kölner Zeitschrift für Soziologie und Sozialpsychologie 52, S. 450-474.

Becker, Rolf (2003): Educational Expansion and Persistent Inequalities of Education: Utilising the Subjective Expected Utility Theory to Explain the Increasing Participation Rates in Upper Secondary School in the Federal Republic of Germany, in: European Sociological Review 19, S. 1-24.

Becker, Rolf (2004): Soziale Ungleichheit von Bildungschancen und Chancengleichheit, in: Rolf Becker / Wolfgang Lauterbach (Hrsg.), Bildung als Privileg? Erklärungen und empirische Befunde zu den Ursachen von Bildungsungleichheiten, Wiesbaden: VS-Verlag für Sozialwissenschaften, S. 161-193.

Bos, Wilfried / Lankes, Eva-Maria / Schwippert, Knut / Valtin, Renate / Voss, Andreas / Badel, Isolde / Plaßmeier Nike (2003): Lesekompetenzen deutscher Grundschülerinnen und Grundschüler am Ende der vierten Jahrgangsstufe im internationalen Vergleich, in: Wilfried Bos et al. (Hrsg.), Erste Ergebnisse aus IGLU. Schülerleistungen am Ende der vierten Jahrgangsstufe im internationalen Vergleich, Münster: Waxmann, S. 69-142.

Boudon, Raymond (1974): Education, Opportunity, and Social Inequality. New York: Wiley.

Breen, Richard / Goldthorpe, John H. (1997): Explaining Educational Differentials. Towards A Formal Rational Action Theory, in: Rationality and Society 9, S. 275-305.

Büchel, Felix / Spieß, C. Katharina / Wagner, Gert (1997): Bildungseffekte vorschulischer Kinderbetreuung, in: Kölner Zeitschrift für Soziologie und Sozialpsychologie 49, S. 528-539. 
Chiswick, Barry R. (1988): Differences in Education and Earnings Across Racial and Ethnic Groups. Tastes, Discrimination and Investment in Child Quality, in: Quarterly Journal of Economics 103, S. 571597.

Cook, Thomas D. / Donald T. Campbell (1979): Quasi-Experimentation. Chicago: Rand McNally.

Diefenbach, Heike / Nauck, Berhard (1997): Bildungsverhalten als 'strategische Praxis': Ein Modell zur Erklärung der Reproduktion von Humankapital in Migrantenfamilien, in: Ludger Pries (Hrsg.), Transnationale Migration. Baden-Baden: Nomos, S. 277-291

Diefenbach, Heike (2004): Bildungschancen und Bildungs(miss)erfolg von ausländischen Schülern oder Schülern aus Migrantenfamilien im System schulischer Bildung, in: Rolf Becker / Wolfgang Lauterbach (Hrsg.), Bildung als Privileg? Wiesbaden: VS-Verlag, S. 225-250.

Entwisle, Doris R. (1995): The Role of Schools in Sustaining Early Childhood Program Benefits, in: Future of Children 5, S. 133-144.

Erikson, Robert / Goldthorpe, John H. (1992): The Constant Flux. A Study of Class Mobility in Industrial Societies, Oxford: Clarendon Press.

Esser, Hartmut (1999): Soziologie. Spezielle Grundlagen. Band 1: Situationslogik und Handeln. Frankfurt am Main: Campus.

Esser, Hartmut (2001): Integration und ethnische Schichtung. MZES-Arbeitspapier Nr. 40. Mannheim: Mannheimer Zentrum für Europäische Sozialforschung.

Gang, Ira N. / Zimmermann, Klaus F. (2000): Is Child Like Parent? Educational Attainment and Ethnic Origin, in: Journal of Human Resources 35, S. 550-569.

Heckhausen, Heinz (1969): Förderung der Lernmotivation und intellektuellen Tüchtigkeiten, in: Deutscher Bildungsrat (Hrsg.), Gutachten und Studien der Bildungskommission: Begabung und Lernen. Stuttgart: Klett, S. 193-228.

Heckman, James J. (1979): Sample Selection Bias as a Specification Error, in: Econometrica 47, S. $153-$ 161.

Hillmert, Steffen / Jacob, Maria (2003): Social inequality in higher education: Is vocational training a pathway leading to or away from university? In: European Sociological Review 19, S. 319-334.

Kreyenfeld, Michaela (2004): Soziale Ungleichheit und Kinderbetreuung. Eine Analyse der sozialen und ökonomischen Determinanten der Nutzung von Kindertageseinrichtungen, in: Rolf Becker / Wolfgang Lauterbach (Hrsg.), Bildung als Privileg? Erklärungen und Befunde zu den Ursachen der Bildungsungleichheit, Wiesbaden: VS-Verlag für Sozialwissenschaften, S. 99-126.

Kreyenfeld, Michaela / Spieß, C. Katharina / Wagner, Gert (2002): Kinderbetreuungspolitik in Deutschland. Möglichkeiten nachfrageorientierter Steuerungs- und Finanzierungsinstrumente, in: Zeitschrift für Erziehungswissenschaft 5, S. 201-221.

Kristen, Cornelia (2002): Hauptschule, Realschule oder Gymnasium? Ethnische Unterschiede am ersten Bildungsübergang, in: Kölner Zeitschrift für Soziologie und Sozialpsychologie 54, S. 534-552.

Lechner, Michael (1998): Mikroökonomische Evaluationsstudien: Anmerkungen zu Theorie und Praxis, in: Friedhelm Pfeiffer / Winfried Pohlmeier (Hrsg.), Qualifikation, Weiterbildung und Arbeitsmarkterfolg. Baden-Baden: Nomos, S. 13-38.

Long, J. Scott (1997): Regression Models for Categorical and Limited Dependent Variables. Thousand Oaks: Sage.

Maddala, Gangadharrao (1978): Selectivity problems in longitudinal data, in: Annales De L'Insee 30-31, S. 423-450.

Mare, Robert D. (1980): Social Background and School Continuation Decisions, in: Journal of the American Stastistical Association 75, S. 295-305.

Mare, Robert D. (1981): Change and Stability in Educational Stratification, in: American Sociological Review 46, S. 72-87.

Nauck, Bernhard (1994): Bildungsverhalten in Migrantenfamilien, in: Peter Büchner / Matthias Grundmann / Johannes Huinink / Lothar Krappmann / Bernhard Nauck / Dagmar Meyer / Sabine Rothe 
(Hrsg.), Kindliche Lebenswelten, Bildung und innerfamiliale Beziehungen. München: Verlag Deutsches Jugendinstitut (DJI), S. 107-142.

Nauck, Bernhard / Diefenbach, Heike / Petri, Kornelia (1998): Intergenerationale Transmission von kulturellem Kapital unter Migrationsbedingungen. Zum Bildungserfolg von Kindern und Jugendlichen aus Migrantenfamilien in Deutschland, in: Zeitschrift für Pädagogik 44, S. 701-722.

Nauck, Bernhard / Diefenbach, Heike (1997): Bildungsbeteiligung von Kindern aus Familien ausländischer Herkunft: Eine methodenkritische Diskussion des Forschungsstandes und eine empirische Bestandsaufnahme, in: Folker Schmidt (Hrsg.), Methodische Probleme der empirischen Erziehungswissenschaft. Hohengehren: Schneider, S. 289-307.

Powell, Justin J. W. / Wagner, Sandra (2001): Daten und Fakten zu Migrantenjugendlichen an Sonderschulen in der Bundesrepublik Deutschland. Berlin: Max-Planck-Institut für Bildungsforschung. Selbständige Nachwuchsgruppe Working Paper 1/2001.

Roßbach, Hans-Günther (2000): Entwicklungen im Kindergarten und in der Grundschule, in: Zeitschrift für Erziehungswissenschaft 3, S. 21-34.

Schütz, Gabriela / Wössmann, Ludger (2005): Wie lässt sich die Ungleichheit der Bildungschancen verringern? In: Ifo-Schnelldienst 21, S. 15-25.

SOEP Group (2001): The German Socio-Economic Panel (GSOEP) after more than 15 years - Overview, in: Elke Holst / Dean R. Lillard / Thomas A. DiPrete (Hrsg.), Proceedings of the 2000 Fourth International Conference of German Socio-Economic Panel Study Users (GSOEP 2000). Vierteljahreshefte zur Wirtschaftsforschung 70, S. 7-14.

Solga, Heike / Wagner, Sandra (2001): Paradoxie der Bildungsexpansion. Die doppelte Benachteiligung von Hauptschülern, in: Zeitschrift für Erziehungswissenschaft 4, S. 107-129.

Spieß, C. Katharina / Büchel, Felix / Wagner, Gert G. (2003): Children's school placement in Germany: does Kindergarten attendance matter? In: Early Childhood Research Quarterly 18, S. 255-270.

Spieß, C. Katharina / Tietze, Wolfgang (2002): Qualitätssicherung in Kindertageseinrichtungen. Gründe, Anforderungen und Umsetzungsüberlegungen für ein Gütesiegel, in: Zeitschrift für Erziehungswissenschaft 5, S. 139-162.

Steinbach, Anja / Nauck, Bernhard (2004): Intergenerationale Transmission von kulturellem Kapital in Migrantenfamilien. Zur Erklärung von ethnischen Unterschieden im deutschen Bildungssystem, in: Zeitschrift für Erziehungswissenschaft 7, S. 20-32.

Wegener, Bernd (1988): Kritik des Prestiges. Opladen: Westdeutscher Verlag.

Weißhuhn, Gernot (2001): Gutachten zur Bildung in Deutschland (http://www.bmbf.de/pub/ gutachten_zur_bildung_in_deutschland.pdf, Stand: August 2003).

Prof. Dr. Rolf Becker

e-mail: rolf.becker@edu.unibe.ch

Patricia Tremel, M. A.

e-mail: patricia.tremel@edu.unibe.ch

Universität Bern

Institut für Erziehungswissenschaft

Abteilung Bildungssoziologie

Muesmattstrasse 27

CH-3012 Bern 